\title{
Octahedral 22 Molecular Geometry
}

National Cancer Institute

\section{Source}

National Cancer Institute. Octahedral 22 Molecular Geometry. NCI Thesaurus. Code C103217.

Octahedral molecular geometry where the Cahn-Ingold-Prelog (CIP) priority of the lowest priority donor atom directly across from (trans to) a priority 1 donor atom is 2 and the priority of the donor atom across from (trans to) the highest priority atom in the plane perpendicular to this reference axis is 2 . 